\title{
Women's perceptions on the effects of COVID-19 on access and use of private sector family planning services in Nigeria: The IntegratE Project
}

The IntegratE Project

Follow this and additional works at: https://knowledgecommons.popcouncil.org/departments_sbsr-rh

Part of the Health Services Research Commons

How does access to this work benefit you? Let us know!

\section{Recommended Citation}

The IntegratE Project. 2021. "Women's perceptions on the effects of COVID-19 on access and use of private sector family planning services in Nigeria: The IntegratE Project," IntegratE Project Brief. Abuja: Population Council. 


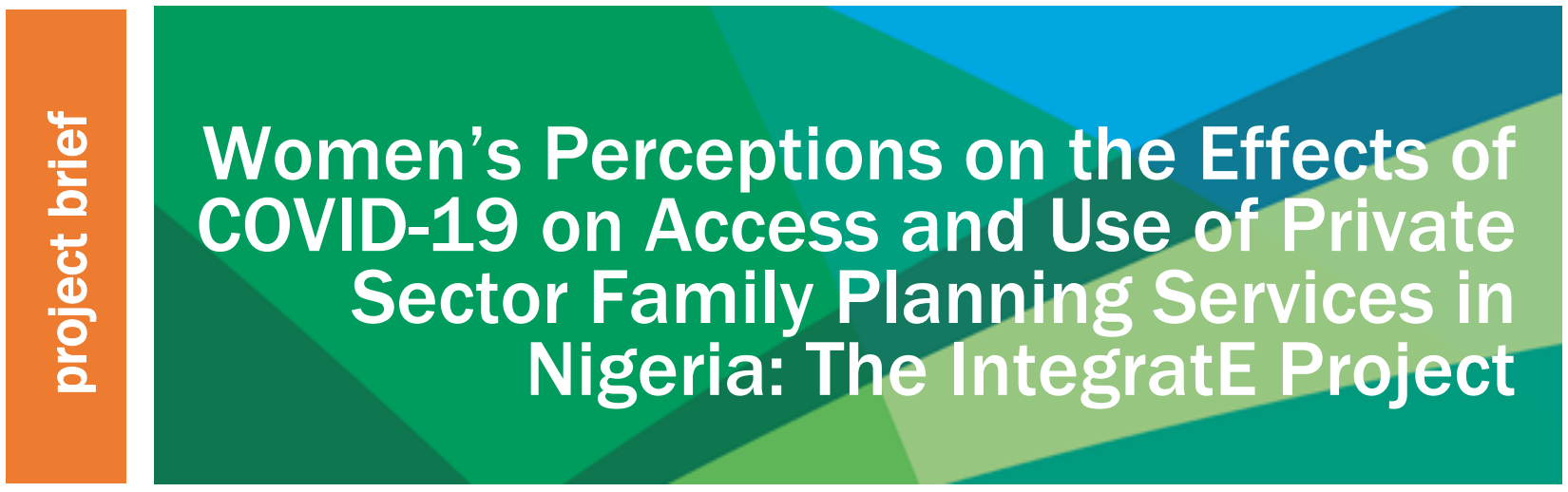

KEY TAKEAWAYS: Family planning clients who received services from Community Pharmacists (CPs) and Patent and Proprietary Medicine Vendors (PPMVs) in Kaduna and Lagos states, appear to have been minimally affected in the early months of the COVID-19 pandemic. Clients reported general continuity of FP services during the pandemic, reinforcing the importance of diverse and accessible outlets to access FP to ensure a sustainable response to health systems shocks.

\section{BACKGROUND}

In Nigeria, Community Pharmacists (CPs) and Patent and Proprietary Medicine Vendors (PPMVs) are the first point of care for many common illnesses within the community. Although CPs and PPMVs are not formally recognized as family planning (FP) service providers, $22 \%$ of modern contraceptive users report receiving their last method from a PPMV and $12 \%$ from a private pharmacy. ${ }^{1}$ PPMVs are especially popular for FP due to their widespread availability, consistent drug stocks, extended hours, personable interactions, and no separate fees for consultations. ${ }^{2,3}$ As the Federal Ministry of Health (FMoH) explores expanding its task sharing policy to include CPs and PPMVs, evidence is needed on an effective regulatory system to support PPMVs and CPs to provide high quality FP services.

As in many places, the COVID-19 pandemic poses unprecedented challenges to health services in Nigeria. By limiting person-to-person contact, especially during initial lock downs, there were concerns regarding disruptions in access to, and use of, FP especially among populations already experiencing high unmet need. ${ }^{4}$ While Nigeria has experienced a lower burden of COVID-19 relative to other countries in Africa and globally, communities across the country faced curfews and other policies restricting gatherings and travel between March and June of 2020. Lagos State remains the epicenter of the nation's pandemic and applied many measures to curtail community transmission including restrictions in movement. Kaduna, a less populous state, recorded concerns in disruptions in access to care and reductions in hospital attendance earlier in the pandemic.

\section{THE INTEGRATE PROJECT}

The IntegratE Project is a four-year initiative (2017-2021) funded by the Bill \& Melinda Gates Foundation and MSD for Mothers ${ }^{5}$ that seeks to increase access to contraceptive methods by involving the 
private sector (CPs and PPMVs) in FP service delivery in Lagos and Kaduna states. IntegratE is implemented by a consortium of partners led by Society for Family Health and includes Marie Stopes International Organization Nigeria, Planned Parenthood Federation of Nigeria, Population Council and PharmAccess. The project seeks to establish a regulatory system with the Pharmacists Council of Nigeria (PCN) to ensure that CPs and PPMVs provide quality FP services, comply with FP regulations, and report service statistics to the Health Management Information System (HMIS).

To achieve this, the IntegratE Project in collaboration with $\mathrm{PCN}$ and the $\mathrm{FMoH}$, is implementing three main activities: (1) a pilot 3-tiered accreditation system for PPMVs based on their healthcare qualifications; (2) a pilot hub-and-spoke supervisory model where CPs (the hub) provide support to PPMVs (spokes) to ensure standard drug stocking practices; and (3) building the capacity of CPs and PPMVs to provide expanded FP services and report service statistics to the HMIS. Under the pilot accreditation system, PPMVs were provided with a standardized FP training to enable them to offer certain FP services based on their tier. CPs function outside of the pilot accreditation system but would receive the same training and provide the same services as Tier 2 PPMVs. The IntegratE Project is simultaneously raising awareness about the FP services that CPs and PPMVs provide. This brief focuses on understanding the impact of the COVID19 pandemic on FP use among women receiving services from $\mathrm{CPs}$ and PPMVs. Additional information on the IntegratE
Project can be found at www.integrateeproject.org.ng.

\section{METHODS}

The IntegratE project conducted a mixedmethods study to assess the effect of a series of interventions on CPs' and PPMVs' capacity to provide high quality FP services in Lagos and Kaduna states.

Researchers adapted an ongoing longitudinal study on client perceptions of FP service delivery to understand effects of the COVID-19 pandemic on fertility intentions and access to sexual and reproductive health services. Yes/no and Likert scale questions related to COIVD-19 were added to interviews conducted with 522 women approximately 9 months after receiving FP services by CPs and PPMVs trained by the IntegratE Project. Data were collected from May to July 2020. Follow up qualitative interviews were conducted with 28 FP users who received FP services from trained CPs and PPMVs in Lagos and Kaduna States. These interviews were conducted by phone between December 2020 and March 2021 after the lockdown had been lifted.

\section{STUDY FINDINGS}

The women interviewed were between 1649 years of age with a mean age of 32 years. About half (51\%) had at least 3 children and many attained at least a secondary education (75\%).

\section{All women were asked about perceptions of COVID-19 risk (Figure 1). Across both states, many women (80\%) agreed that they would know what to do if}


Figure 1: Client's perceived risk of COVID-19 $(n=522)$

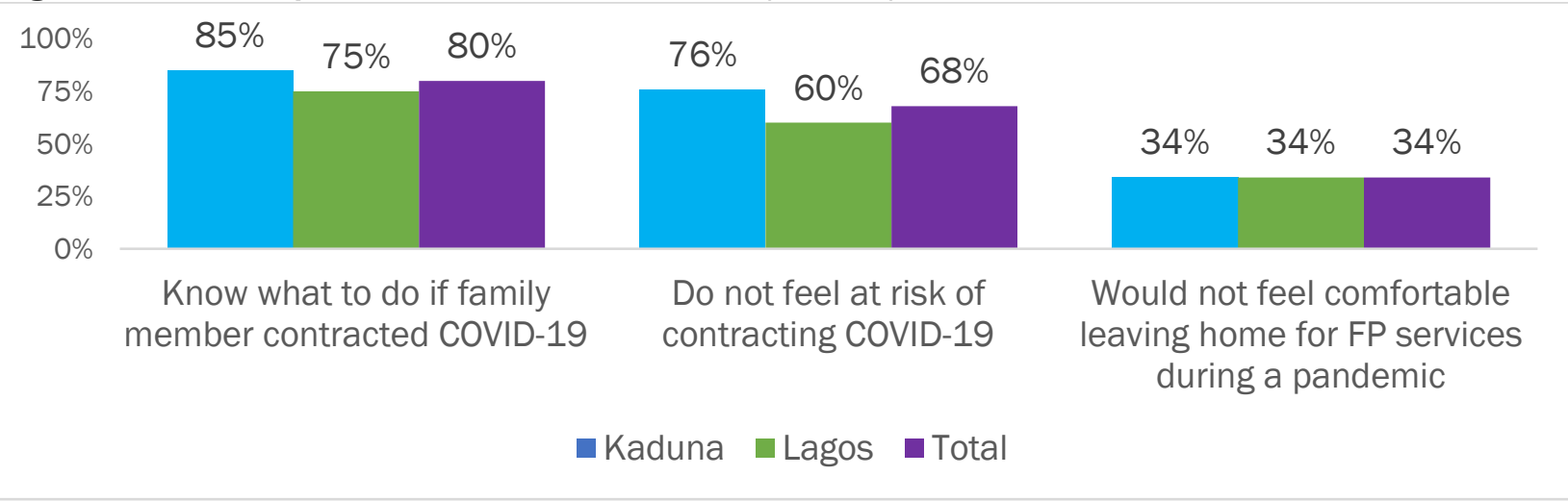

Figure 2: Contraceptive use at 6 months $(n=479)$

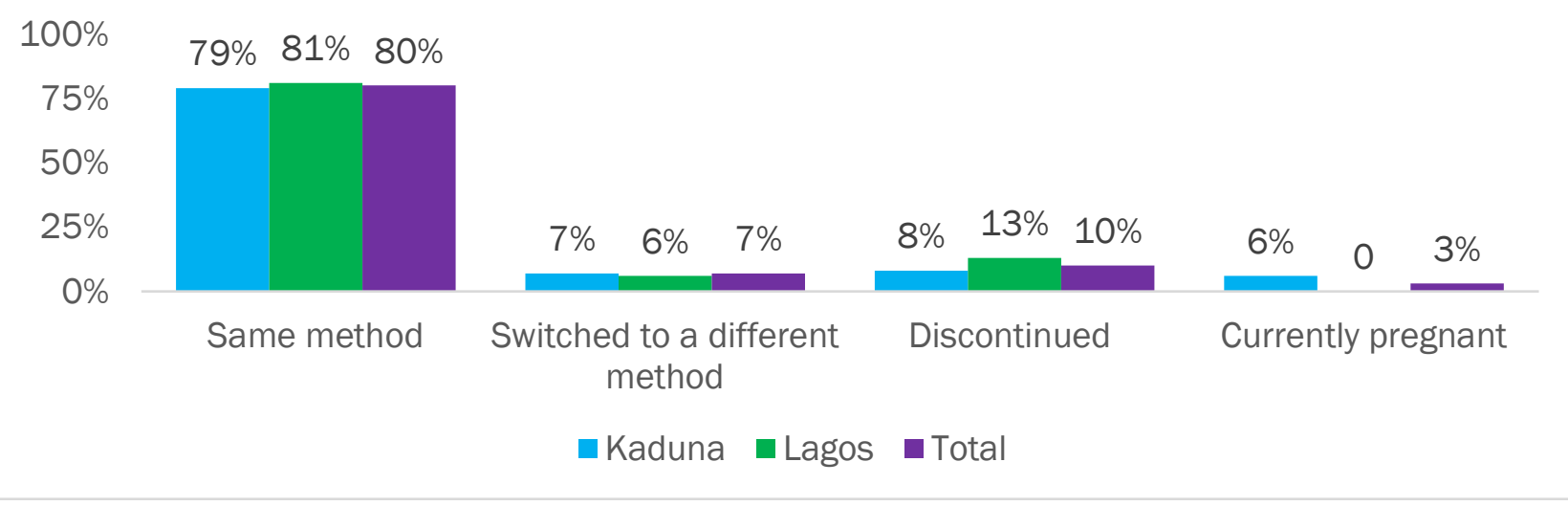

someone became ill with COVID-19 and $69 \%$ did not report feeling at risk of contracting the virus. About one-third (34\%) of woman, however, agreed that they did not feel comfortable leaving their house for FP services.

Few women reported that COVID-19 had any influence on their fertility intentions (data not shown). Only $8 \%$ reported wanting to delay having another child, and $8 \%$ reported wanting fewer children. The perceived influence of COVID-19 on fertility intentions was higher in Kaduna state than in Lagos.

Despite the pandemic and associated restrictions, most women reported that they were still using FP (Figure 2). Over $80 \%$ were using the same FP method that they had received approximately 9 months prior either directly from an IntegratE CP or PPMV or by referral, and $7 \%$ had switched to a different method. Ten percent reported that they had stopped using a FP method. Among the 437 women still using FP methods, few reported that COVID delayed them in accessing services (3\%) or getting a resupply of FP commodities (6\%) (data not shown).

In the qualitative follow-up interviews, FP users indicated that COVID-19 did not impact their FP behaviors. When asked by the interviewer if COVID-19 had impacted their decision to continue to use FP, an injectable user replied: "Nothing changed in that aspect" 
-28-year-old married woman with secondary education and two children from Kaduna state

An implant user shared her views about why some may not want to go to the hospitals to get FP, including herself, during the pandemic because of the potential COVID-19 presence at the hospital. She described it as follows:

"...the only way it [FP use] can be affected is that, why you will be scared is that where you want to do it [access FP], if it's a hospital that there are a lot of people, you won't be able to go during that time because my husband is someone that doesn't take the Covid issue lightly and he is careful as he believes in it strongly more than me. He travels outside the country so he knows what obtains there so if it's a place there will be a lot of people like the hospital, I might not go during that time"

-42-year-old married implant user with two children and secondary education; Lagos state)

In the follow-up qualitative interviews, FP users also highlighted the economic impact COVID-19 has had on their livelihoods. The following quote illustrates the views expressed by a woman hit hard by the lockdown:

"Truthfully everything stopped - the way you get money, the children stopped going to school and presently, the business itself has stopped."

-28-year-old married injectable user with one child and secondary education; Kaduna state

\section{CONCLUSIONS \& IMPLICATIONS}

The ongoing COVID-19 pandemic in Lagos and Kaduna has had limited influence among women who received FP services from CPs and PPMVs. While these results were collected soon after the pandemic began, follow-up interviews with FP users confirmed that the pandemic had not influenced their FP behaviors several months after the lockdowns had concluded, though COVID-19 was still present at a low infection rate.

Community-based providers, such as PPMVs and CPs served as a continuous source of FP, reinforcing the importance of promoting diverse and accessible service delivery options for FP access and a resilient FP environment in Nigeria.

However, state-level lockdowns related to COVID-19 disrupted livelihoods and led to lasting economic impacts. As the pandemic continues to evolve with the introduction of new variants and the availability of vaccines, women's perceptions of COVID-19 and their FP behaviors may also change. Given this context, additional studies are needed to assess changing perception of COVID-19 and FP use, in addition to the availability of FP services.

\section{References}

1. National Population Commission (NPC) [Nigeria] and ICF. 2019. Nigeria Demographic and Health Survey 2018. Abuja, Nigeria, and Rockville, Maryland, USA: NPC and ICF.

2. Brugha R, Zwi A (2002) "Improving the quality of private sector delivering of public health services: challenges and strategies." Health Policy Plan, 13:103-120.

3. Adetunji JA (1991) "Response of parents to five killer diseases among children in a Yoruba community,

Nigeria." Social Science Medicine 32:1379-1387.

4. UNFPA. 2021. Impact of COVID-19 on Family

Planning: What we know one year into the pandemic. https://www.unfpa.org/sites/default/files/resourcepdf/COVID Impact FP V5.pdf

5. This program is co-funded by, developed and is being implemented in collaboration with MSD for Mothers, MSD's $\$ 500$ million initiative to help create a world where no woman dies giving life. MSD for Mothers is an initiative of Merck \& Co., Inc., Kenilworth, NJ, U.S.A. 